\title{
Bnip3 as a Dual Regulator of Mitochondrial Turnover and Cell Death in the Myocardium
}

\author{
Åsa B. Gustafsson
}

Received: 8 December 2010/ Accepted: 15 December 2010/Published online: 6 January 2011

(C) The Author(s) 2011. This article is published with open access at Springerlink.com

\begin{abstract}
The Bcl-2 adenovirus E1B 19 kDa-interacting protein 3 (Bnip3) is a pro-apoptotic BH3-only protein associated with the pathogenesis of many diseases, including cancer and cardiovascular disease. Studies over the past decade have provided insight into how Bnip3 induces mitochondrial dysfunction and subsequent cell death in cells. More recently, Bnip3 was identified as a potent inducer of autophagy in cells. However, the functional role of Bnip3-mediated autophagy has been difficult to define and remains controversial. New evidence has emerged suggesting that Bnip3 is an important regulator of mitochondrial turnover via autophagy in the myocardium. Also, studies suggest that the induction of Bnip3-dependent mitochondrial autophagy is a separately activated process independent of Bax/Bak and the mitochondrial permeability transition pore (mPTP). This review discusses the current understanding of the functional role that Bnip3 plays in the myocardium. Recent studies suggest that Bnip3 might have a dual function in the myocardium, where it regulates both mitochondrial turnover via autophagy and cell death and that these are two separate processes activated by Bnip3.
\end{abstract}

Keywords Apoptosis - Autophagy - Bcl-2 family · Bnip3 $\cdot$ Mitochondria $\cdot$ Necrosis

Contracting cardiac myocytes requires large amounts of energy, which is provided by mitochondria through

\footnotetext{
Å. B. Gustafsson $(\bowtie)$

Skaggs School of Pharmacy and Pharmaceutical Sciences, University of California, San Diego, 9500 Gilman Drive MC 0758, La Jolla, CA 92093-0758, USA

e-mail: asag@ucsd.edu
}

oxidative phosphorylation. Due to this large demand for adenosine triphosphate (ATP), cardiac myocytes have the highest mitochondria volume density of all the cells in the body.

Although the primary function of mitochondria is to meet the high energy demand of the beating heart, they also are important regulators of cell death. The Bcl-2 proteins display either anti- or pro-apoptotic function [17] and control mitochondrial membrane permeabilization. They are responsible for monitoring changes in the intracellular environment. Anti-apoptotic members such as Bcl-2, $\mathrm{Bcl}-\mathrm{X}_{\mathrm{L}}$, and Mcl-1 protect mitochondrial integrity, whereas pro-apoptotic proteins promote the release of pro-death proteins from the mitochondria.

The pro-apoptotic proteins can be divided further into the BH3-only proteins including Bid, Bad, Bim, and their effectors Bax and Bak. The BH3-only proteins are activated in response to stress, such as DNA damage or serum deprivation, and then transduce the signal to Bax, Bak, or both. At least 10 different $\mathrm{BH} 3$-only proteins have been identified, and their activity is regulated by transcription, posttranslational modification, or both [17].

Bcl-2/adenovirus E1B 19-kilodalton interacting protein (Bnip3) and its homologue, Nix (also called Bnip3L), are pro-apoptotic $\mathrm{BH} 3$-only proteins that play key roles in the pathogenesis of many diseases such as heart failure $[40,55]$ and cancer [5]. Bnip3 and Nix have several features in common with the $\mathrm{BH} 3$-only proteins of the Bcl-2 family. They both contain BH3 domains [20, 52], localize to the mitochondrial membrane $[6,7,19,52]$, activate downstream effectors Bax/Bak [8, 27], and interact with antiapoptotic Bcl-2 and Bcl- $\mathrm{X}_{\mathrm{L}}$ [20, 39].

However, Bnip3 and Nix have a number of features that set them apart from other BH3-only proteins such as tBid and Bad. For instance, the transmembrane domains, but not 
the BH3 domains of Bnip3 and Nix, play a major role in the induction of cell death [7]. Bnip3 and Nix also are weak inducers of cell death compared with other BH3-only proteins, and they induce opening of the mitochondrial permeability transition pore (mPTP) [20, 40, 50]. Moreover, they are important regulators of mitochondrial autophagy [19, 38, 43, 56].

Autophagy is an important cellular process involved in the degradation of long-lived proteins and organelles [33]. This process occurs constitutively in most cells but is rapidly upregulated when a change in the cellular environment occurs such as a decrease in nutrients.

Early studies suggested that autophagy was a nonselective process in which cytoplasmic material was randomly sequestered by an autophagosome and subsequently delivered to the lysosome, where it was degraded by lysosomal proteases [33]. However, it now is clear that this process is selective and that organelles such as mitochondria and endoplasmic reticulum (ER) can be specifically removed while other structures are left intact.

Recent studies have implicated both Bnip3 and Nix as important regulators of mitochondria autophagy. This review discusses recent advances in understanding the functional role of Bnip3 in the myocardium. Recent studies suggest that Bnip3 might have dual functions in the myocardium, where it regulates both mitochondrial turnover via autophagy and cell death.

\section{Transcriptional Regulation of Bnip3}

Early studies found that Bnip3 was not expressed in most cells and was subject to transcriptional regulation. Several investigations showed that hypoxia leads to upregulation of Bnip3 in cells, including neonatal myocytes [4, 40]. The Bnip3 promoter contains a hypoxia-inducible factor-1 (HIF-1)-responsive element (HRE) and is potently activated by both hypoxia and forced expression of HIF-1 $\alpha$ [4, 47].

Yurkova et al. [54] also reported that Bnip3 is a direct transcriptional target of E2F-1 and involved in E2F-1induced cell death. Because significant upregulation of Bnip3 leads to mitochondrial dysfunction and cell death [27, 40, 50], it clearly is important for cells to suppress expression of Bnip3 under normal conditions.

Recent work from the Kirshenbaum group has demonstrated a critical role of $\mathrm{NF}-\kappa \mathrm{B}$ in suppressing Bnip3 expression in neonatal cardiac myocytes [44]. Interestingly, these researchers found that cells derived from p65 knockout mice or myocytes with defective NF- $\kappa \mathrm{B}$ signaling exhibited increased basal Bnip3 gene expression and cell death, suggesting that $\mathrm{NF}-\kappa \mathrm{B}$ has an important survival role in myocytes. In addition, they demonstrated that $\mathrm{NF}-\kappa \mathrm{B}$ recruited histone deacetylase-1 (HDAC-1) to the Bnip3 promoter, providing a mechanism for transcriptional repression of Bnip3 and cell death under basal conditions [45].

\section{Posttranslational Regulation of Bnip3}

Although many cells suppress Bnip3 expression under normal conditions, some tissues and cell lines constitutively express Bnip3. For instance, Galvez et al. [14] found high levels of the Bnip3 transcript in heart, liver, and kidney but low or undetectable levels in brain, spleen, and lung tissue. Similarly, Bruick [4] found that Bnip3 was expressed in $\mathrm{CHO}-\mathrm{K} 1$ and HepG2 cells but was barely detectable in Rat-1 and CV-1 cells. Bnip3 is not expressed in neonatal cardiac myocytes under normal conditions $[16,54]$ but is constitutively expressed in adult hearts and to a lower level in HL-1 myocytes [11, 19]. This suggests that Bnip3 is maintained in an inactive state under normal conditions and regulated by posttranslation modification.

The fact that Bnip3 must be subjected to additional activation is supported by the fact that adult cardiac myocytes are remarkably resistant to Bnip3 under normal conditions. Diwan et al. [11] found that even a 50-fold increase in Bnip3 expression in hearts from transgenic mice produced low levels of apoptosis. However, apoptosis of cardiac myocytes was significantly increased in Bnip3 transgenic mouse hearts compared with wild type hearts after myocardial infarction, suggesting that ischemia induced activation of Bnip3.

Bnip3 contains a transmembrane domain at its C-terminus, which is important for mitochondrial targeting, homodimerization, and pro-apoptotic activity [6, 50]. Deletion of the transmembrane domain completely abrogates homodimerization and cell death [6].

We found that the $\mathrm{N}$-terminus contains a conserved cysteine residue, which also is important for Bnip3 homodimerization and pro-death activity. Bnip3 is anchored in the mitochondrial outer membrane via its C-terminal transmembrane domain, whereas the cysteine residue in the $\mathrm{N}$-terminus is facing the cytosol, which makes it susceptible to oxidation [50].

We also found that Bnip3 functions as a redox sensor of oxidative stress, with increased oxidative stress in the myocardium in response to ischemia/reperfusion (I/R) resulting in oxidation of the $\mathrm{N}$-terminal cysteine residue in Bnip3 and promotion of homodimerization [28]. When the cysteine is mutated to an alanine, homodimerization of Bnip3 is reduced, which correlates with reduced cell death. Thus, these studies suggest that Bnip3 functions as a redox sensor, with increased oxidative stress inducing homodimerization and activation of Bnip3 via cooperation of the N-terminal cysteine residue and the C-terminal transmembrane domain. 
In addition, Bnip3 contains several potential phosphorylation sites, and studies have reported that Bnip3 activity is regulated by phosphorylation. Graham et al. [15] reported that hypoxia-reoxygenation induced phosphorylation of Bnip3 and that elevated levels of phosphorylated Bnip3 correlated with increased cell death. Similarly, Mellor et al. [34] reported that treatment of cells with microtubule inhibitors resulted in the phosphorylation of Bnip3 and that the phosphorylation of Bnip3 increased its stability. It is currently unknown whether and how phosphorylation regulates Bnip3 activity and which kinase or kinases are responsible for the phosphorylation.

\section{Bnip3 Induces Mitochondrial Dysfunction via Bax/Bak and the mPTP}

Bnip3 is primarily located in the mitochondria, where it is integrated into the mitochondrial membrane [19]. It is well documented that overexpression of Bnip3 leads to loss of mitochondrial membrane potential and cell death [27, 40, 50]. However, the exact mechanism by which Bnip3 perturbs mitochondrial function still is controversial. Permeabilization of the mitochondrial outer membrane can occur via two different mechanisms. It can be induced via activation of Bax/Bak, which forms pores in the mitochondrial outer membrane that accommodate passage of proteins in the intermembrane space, such as cytochrome $c$, to the cytosol [25, 29].

Alternatively, loss of outer membrane integrity can occur via opening of the MPTP, a nonspecific pore in the inner mitochondrial membrane that is permeable to molecules smaller than $1.5 \mathrm{kDa}$ [18]. Opening of the mPTP results in swelling of the inner membrane and subsequent rupture of the outer mitochondrial membrane. The rupture causes release of all intermembrane space proteins into the cytosol $[18,23]$. The mPTP is thought to mainly play a role in necrosis, whereas the $\mathrm{Bax} / \mathrm{Bak}$ channel is involved in apoptotic cell death. Interestingly, Bnip3 is reported to mediate mitochondrial dysfunction via opening of the mPTP [26, 40, 50] and via activation of Bax/Bak [27] (Fig. 1).

All BH3-only proteins known to date promote activation of Bax, Bak, or both [51, 57], which in turn causes cytochrome $c$ release, caspase activation, and apoptosis [31]. In our studies, we found that Bnip3 induces release of cytochrome $c$ from mitochondria in a Bax/Bak-dependent manner and that cells lacking Bax/Bak are resistant to Bnip3-mediated cell death [27, 37]. Findings have shown that the presence of the caspase inhibitor $\mathrm{z}-\mathrm{VAD}$-fmk can suppress Bnip3-mediated cell death in neonatal cardiac myocytes [40], HL-1 cells [19], and HeLa cells [30].

Many other studies have reported that Bnip3 can mediate mitochondrial dysfunction and cell death via opening of the mPTP $[26,27,40,50]$. In one of the first studies, Bnip3 was found to induce rapid opening of the mPTP and cell death in $293 \mathrm{~T}$ cells, which could be reduced in the presence of cyclosporine A (CsA) or bongkrekic acid (BA), two different inhibitors of the mPTP [50]. Interestingly, this study found that maximum suppression of cell death was only $50 \%$ with either inhibitor, suggesting that Bnip3 mediated cell death by two separate mechanisms in these cells.

In our studies, we found that although Bnip3 can induce opening of the mPTP, it is not required for Bnip3-mediated cell death [27] and that inhibiting the MPTP had no effect on Bax/Bak activation in response to Bnip3. In addition, we recently discovered that Bnip3 can induce permeabilization of the inner mitochondrial membrane, which results in large-amplitude swelling of the inner membrane via a mechanism independent of the mPTP. Other BH3-only proteins such as tBid do not induce permeabilization of the outer membrane. The Bnip3 homologue Nix has been shown to induce cell death via activation of Bax/Bak as well as via opening of the MPTP [8], but it is currently unknown whether Nix, like Bnip3, induces permeabilization of the inner membrane. Exactly how Bnip3 induces permeabilization of the inner membrane currently is unknown, and further studies are needed to elucidate the exact mechanism or mechanisms by which Bnip3 mediates mitochondrial dysfunction.

\section{Bnip3 and Mitochondrial Dynamics}

We and others have found that overexpression of Bnip3 induces mitochondrial fragmentation or fission in cells $[19,30]$. Mitochondria are highly dynamic organelles that constantly undergo fission to produce smaller organelles or fusion to produce tubular mitochondrial networks. Mitochondrial fission has been implicated in apoptosis [13] and also contributes to myocardial I/R injury [36]. Moreover, findings have shown that mitochondrial fission is essential for the removal of mitochondria by autophagy and that inhibition of fission results in disruption of mitochondrial autophagy and accumulation of dysfunctional mitochondria [10, 49].

Mitochondrial dynamics are determined by the balance between fusion and fission events, which are known to be regulated by at least four dynamin-related GTPases. Mitochondrial fission is regulated by Drp1 and hFis1 [46, 53], whereas fusion requires mitofusins 1 and 2 (mfn 1 and mnf2) in the outer membrane and Opal in the inner membrane [9, 24]. We found that overexpression of Bnip3 induced translocation of Drp1 to mitochondria, which correlated with fragmentation of mitochondria in both fibroblasts and myocytes [27] (unpublished data). 


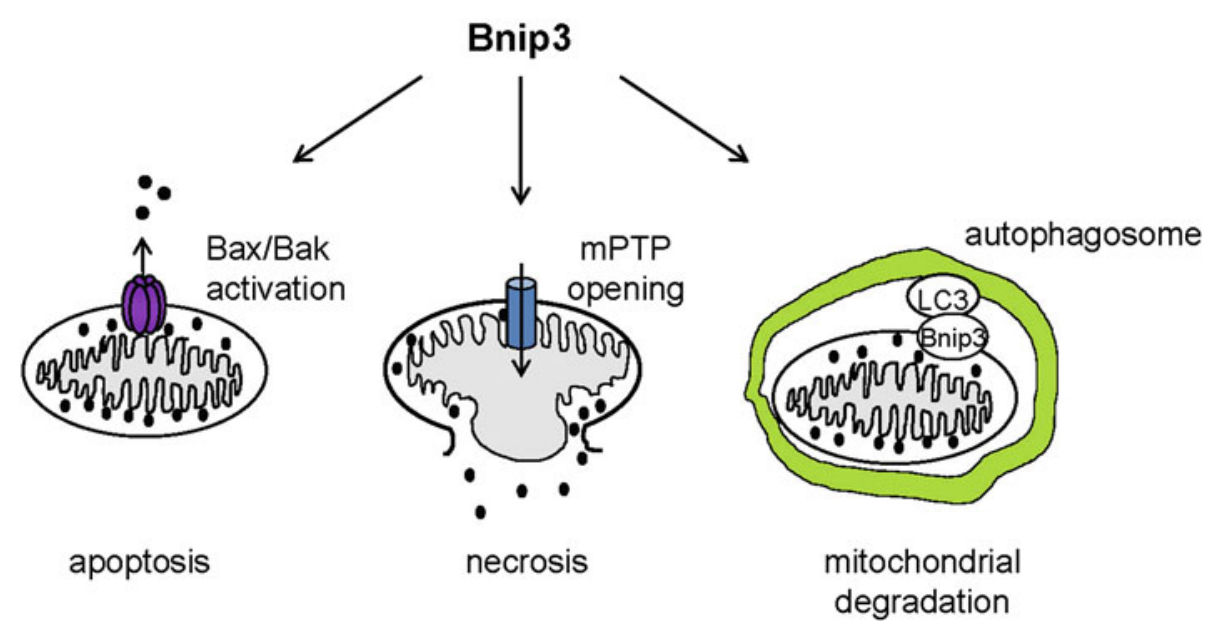

Fig. 1 The atypical BH3-only protein Bnip3 regulates cell death of cardiac myocytes through two separate pathways involving the mitochondria and promotes mitochondrial turnover via autophagy. In response to stress, Bnip3 activates the intrinsic apoptotic pathway via Bax and Bak. Activation of Bax/Bak causes permeabilization of the outer mitochondrial membrane and release of pro-apoptotic proteins such as cytochrome $c$, leading to activation of caspases and cell death. Bnip3 also can induce opening of the mitochondrial permeability transition pore (mPTP), a nonselective channel permeable to

Similarly, Landes et al. [30] reported that Bnip3-mediated mitochondrial fission occurred before cytochrome $c$ release and that fission was inhibited by the dominant negative Drp1K38A in HeLa cells. The same study also found that Bnip3 interacted with the fusion protein Opa1, suggesting that Bnip3 mediates mitochondrial fission by inhibiting fusion. However, this study did not assess whether Bnip3-mediated cell death was affected when fission was inhibited. Thus, the functional significance of Bnip3mediated fission still is unclear, and it is unknown whether it contributes to cell death or plays a role in mitochondrial autophagy. Interestingly, mitochondrial fission has been shown to produce two mitochondrial fragments with different $\Delta \psi \mathrm{m}$, and the fragments with low $\Delta \psi \mathrm{m}$ usually were removed by autophagy [49]. Thus, it is possible that Bnip3mediated mitochondrial fragmentation is required for mitochondrial autophagy.

\section{Bnip3 Regulates Mitochondrial Turnover via Autophagy}

Although Bnip3 is considered a pro-apoptotic protein, many studies have reported that Bnip3 also is a potent inducer of autophagy in many different cell types, including cardiac myocytes $[1,3,19,22,38,41]$. However, the functional role of Bnip3-mediated autophagy is controversial and reportedly both causes and protects against cell death. molecules smaller than $1.5 \mathrm{kDa}$. Opening of the mPTP leads to swelling of the inner mitochondrial membrane and subsequent rupture of the outer membrane. Bnip3 also promotes mitochondrial turnover, in which Bnip3 targets mitochondria for removal by autophagosomes. Bnip3 is anchored in the outer mitochondrial membrane via its C-terminal transmembrane domain, where it can interact with LC3/ GAPARAP on the autophagosome, thereby anchoring the damaged mitochondrion to the autophagosome

In our studies, we found that inhibiting autophagy enhanced Bnip3-mediated cell death in HL-1 cells, whereas enhancing autophagy reduced cell death, suggesting that autophagy is a protective process [19]. We recently found that Bnip3 induced autophagy in apoptosis-resistant cells lacking Bax/Bak and that inhibiting autophagy resulted in necrotic cell death [41]. Similarly, Bellot et al. [3] found that Bnip3-mediated autophagy during hypoxia was a survival mechanism that promoted tumor progression. In contrast, Bnip3 contributed to autophagic cell death during hypoxia [1] and induced autophagic cell death in malignant glioma cells [22].

Autophagy is important for the removal of excess or damaged organelles, including mitochondria [33]. Recent studies have demonstrated that both Bnip3 and Nix induce removal of mitochondria via autophagy in cells. In fact, Schweers et al. [43] found that Nix was essential for mitochondrial clearance via autophagy during reticulocyte maturation.

We also found that Bnip3 induced mitochondrial autophagy in cardiac myocytes $[19,38]$. We initially hypothesized that autophagy was upregulated in response to Bnip3 for removal of damaged mitochondria [19]. However, evidence now shows that the induction of mitochondrial autophagy is specifically activated by Bnip3 and Nix and that this process is separate from activation of cell death (Fig. 1).

We also found that almost all the autophagosomes contain mitochondria in myocytes overexpressing Bnip3 

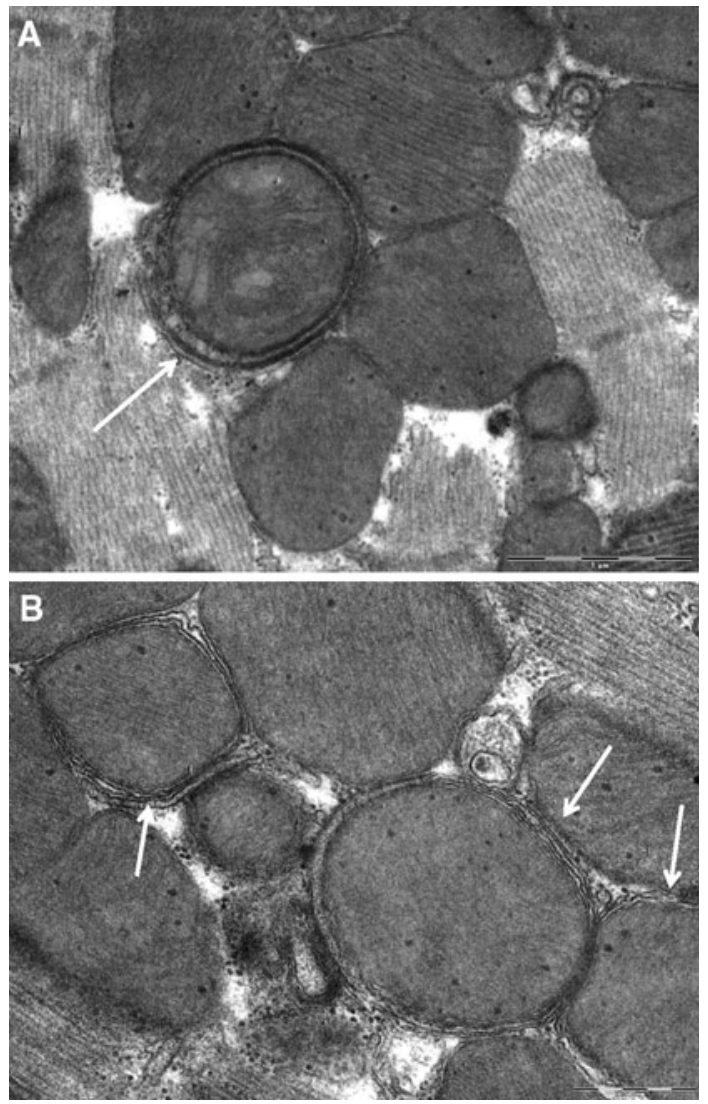

Fig. 2 Ultrastructural analysis of adult cardiac myocytes overexpressing Bnip3 shows extensive induction of mitochondrial autophagy. Freshly isolated cells were infected with an adenovirus encoding Bnip3 for $48 \mathrm{~h}$ and then fixed for analysis. Electron micrographs showed the presence of autophagosomes containing mitochondria (white arrows). a Scale bar $=1 \mu \mathrm{m} . \mathbf{b}$ Scale bar $=500 \mathrm{~nm}$

(Fig. 2), suggesting that Bnip3 specifically induces mitochondrial autophagy and not "nonselective" autophagy [38]. Bnip3-overexpressing myocytes were remarkably resistant to Bnip3 overexpression, and although myocytes had a substantial amount of their mitochondria removed by autophagy, they still were viable [38].

Furthermore, opening of the mPTP has been implicated in promoting selective removal of damaged mitochondria by autophagosomes [32]. However, we found that treatment with the MPTP inhibitor CsA did not inhibit mitochondrial autophagy in cardiac myocytes [38]. In addition, cyclophilin D (cypD) is an essential component of the mPTP [2], and we found that Bnip3 induced autophagy to the same extent in fibroblasts isolated from wild type and cypD-deficient mice, suggesting that Bnip3-mediated autophagy is not dependent on opening of the MPTP.

We also found that Bnip3 induces mitochondrial autophagy in Bax/Bak-null fibroblasts, suggesting that Bnip3 induces this process even in the absence of mitochondrial permeabilization and apoptosis [41]. Schweers et al. [43] demonstrated that Nix-dependent mitochondrial clearance was normal in Bax/Bak-null reticulocytes and that mitochondrial clearance was unaffected by inhibitors of the mPTP in wild-type reticulocytes. Thus, these data suggest that induction of Bnip3- and Nix-dependent mitochondrial autophagy is a separately activated process independent of Bax/Bak and the mPTP.

Studies from Dr. Gerald Dorn's lab have confirmed that both Bnip3 and Nix have alternate and overlapping functions as regulators of mitochondrial turnover in the adult myocardium. Studies investigating transgenic mice overexpressing Bnip3 or Nix in the myocardium have shown that the adult hearts are remarkably resistant to cell death. Using inducible transgenic mice, these studies found that increased expression of Nix or Bnip3 in adult hearts did not cause functionally significant cardiomyocyte apoptosis in adult mice $[11,48]$. Interestingly, enhanced expression of Bnip3 or Nix in neonatal mice caused progressive apoptotic cardiac dilation.

These studies suggest that Bnip3 and Nix might have a function different from promoting apoptosis in the adult heart. Moreover, long-term studies of mice deficient in Nix, Bnip3, and both Bnip3 and Nix have shown that hearts of aged knockout mice accumulate dysfunctional mitochondria and that this occurs twice as fast in hearts lacking both Bnip3 and Nix [12].

Moreover, the accumulation of dysfunctional mitochondria correlated with development of cardiac dysfunction. Nix-null mice experienced cardiac enlargement and reduced ventricular contractility at 60 weeks, whereas the Bnip3/Nix double-knockout mice showed development of the same cardiac phenotype as the Nix-null mice at 30 weeks of age. These data suggest that the "pro-death" Bnip3 and Nix have an alternative but overlapping function in the adult heart, where they regulate mitochondrial turnover via autophagy.

\section{Bnip3 and Nix as Potential Autophagy Receptors}

Exactly how Bnip3 and Nix induce specific removal of mitochondria via autophagy is unknown. However, two separate investigations recently reported that Nix interacts with the autophagy proteins LC3 and GABARAP [35, 42]. Novak et al. [35] also found that Nix recruited GABARAP to depolarized mitochondria. GABARAP belongs to a family of small ubiquitin-like adaptor proteins implicated in cellular trafficking of the $\mathrm{GABA}_{\mathrm{A}}$ receptor and in intracellular autophagy. Findings have shown that GABARAP co-localizes with LC3-II on the autophagosomal membrane [21].

Both studies identified a potential LC3-interacting region in the N-terminal domain of Nix. They also found that the tryptophan residue in this region was essential in interaction with GABARAP/LC3 and that mutation of this 
residue disrupted the interaction between Nix and GABARAP/LC3 [35, 42] and inhibited mitochondrial clearance [35].

Interestingly, the LC3-interacting region and the tryptophan residue are conserved between Nix and Bnip3, suggesting that Bnip3 likely functions similar to Nix as a receptor for proteins on the forming autophagosomal membrane. The interaction between Nix/Bnip3 and LC3/ GABARAP would tether the mitochondrion to the autophagosome.

In our studies, we discovered that Bnip3 also interacts with LC3 [41]. However, further studies are needed to determine whether Bnip3 interacts with GABARAP/LC3 via its N-terminus. Because both Nix and Bnip3 function as dimers, it also will be interesting to determine whether dimerization via the transmembrane domain is essential for the interaction with GABARAP/LC3.

\section{Conclusions}

Studies suggest that both Bnip3 and Nix have dual roles in the myocardium: to activate cell death and to induce mitochondrial turnover via autophagy (Fig. 1). The ability of Bnip3 and Nix to induce cell death and mitochondrial autophagy appears to be two independent functions. This therefore raises the question of when they induce mitochondrial autophagy and when they activate cell death. It is tempting to speculate that under normal conditions, Bnip3 and Nix promote turnover of old dysfunctional mitochondria, possibly through their ability to reduce mitochondrial membrane potential.

In contrast, in response to stress such as hypoxia or pressure overload, when mitochondria are severely damaged and the cell is beyond rescue, Bnip3 and Nix are elevated to levels at which they activate the cell death program instead. Because Bnip3 has been implicated in the pathogenesis of heart failure, Bnip3 represents an important potential therapeutic target for treatment or prevention of disease. However, based on new evidence suggesting that Bnip3 has an alternate function in the cell, inhibiting Bnip3 may lead to accumulation of dysfunctional mitochondria and induction of cardiac dysfunction. Further studies are needed to determine when and how Bnip3 switches between the two functions in the heart.

Acknowledgments Åsa B. Gustafsson is supported by a Scientist Development Grant from the American Heart Association and by NIH grants R01HL087023, R01HL101217, and R01HL092136.

Open Access This article is distributed under the terms of the Creative Commons Attribution Noncommercial License which permits any noncommercial use, distribution, and reproduction in any medium, provided the original author(s) and source are credited.

\section{References}

1. Azad MB, Chen Y, Henson ES, Cizeau J, McMillan-Ward E, Israels SJ, Gibson SB (2008) Hypoxia induces autophagic cell death in apoptosis-competent cells through a mechanism involving BNIP3. Autophagy 4:195-204

2. Baines CP, Kaiser RA, Purcell NH, Blair NS, Osinska H, Hambleton MA, Brunskill EW, Sayen MR, Gottlieb RA, Dorn GW, Robbins J, Molkentin JD (2005) Loss of cyclophilin D reveals a critical role for mitochondrial permeability transition in cell death. Nature 434:658-662

3. Bellot G, Garcia-Medina R, Gounon P, Chiche J, Roux D, Pouyssegur J, Mazure NM (2009) Hypoxia-induced autophagy is mediated through hypoxia-inducible factor induction of BNIP3 and BNIP3L via their BH3 domains. Mol Cell Biol 29: 2570-2581

4. Bruick RK (2000) Expression of the gene encoding the proapoptotic Nip3 protein is induced by hypoxia. Proc Natl Acad Sci USA 97:9082-9087

5. Burton TR, Gibson SB (2009) The role of Bcl-2 family member BNIP3 in cell death and disease: NIPping at the heels of cell death. Cell Death Differ 16:515-523

6. Chen G, Ray R, Dubik D, Shi L, Cizeau J, Bleackley RC, Saxena S, Gietz RD, Greenberg AH (1997) The E1B 19K/Bcl-2-binding protein Nip3 is a dimeric mitochondrial protein that activates apoptosis. J Exp Med 186:1975-1983

7. Chen G, Cizeau J, Vande VC, Park JH, Bozek G, Bolton J, Shi L, Dubik D, Greenberg A (1999) Nix and Nip3 form a subfamily of pro-apoptotic mitochondrial proteins. J Biol Chem 274:7-10

8. Chen Y, Lewis W, Diwan A, Cheng EH, Matkovich SJ, Dorn GW II (2010) Dual autonomous mitochondrial cell death pathways are activated by Nix/BNip3L and induce cardiomyopathy. Proc Natl Acad Sci USA 107:9035-9042

9. Cipolat S, Martins de Brito O, Dal Zilio B, Scorrano L (2004) OPA1 requires mitofusin 1 to promote mitochondrial fusion. Proc Natl Acad Sci USA 101:15927-15932

10. Dagda RK, Cherra SJ III, Kulich SM, Tandon A, Park D, Chu CT (2009) Loss of PINK1 function promotes mitophagy through effects on oxidative stress and mitochondrial fission. J Biol Chem 284:13843-13855

11. Diwan A, Krenz M, Syed FM, Wansapura J, Ren X, Koesters AG, Li H, Kirshenbaum LA, Hahn HS, Robbins J, Jones WK, Dorn GW (2007) Inhibition of ischemic cardiomyocyte apoptosis through targeted ablation of Bnip3 restrains postinfarction remodeling in mice. J Clin Invest 117:2825-2833

12. Dorn GW II (2010) Mitochondrial pruning by Nix and BNip3: an essential function for cardiac-expressed death factors. J Cardiovasc Transl Res 3:374-383

13. Frank S, Gaume B, Bergmann-Leitner ES, Leitner WW, Robert EG, Catez F, Smith CL, Youle RJ (2001) The role of dynaminrelated protein 1, a mediator of mitochondrial fission, in apoptosis. Dev Cell 1:515-525

14. Galvez AS, Brunskill EW, Marreez Y, Benner BJ, Regula KM, Kirschenbaum LA, Dorn GW II (2006) Distinct pathways regulate proapoptotic Nix and BNip3 in cardiac stress. J Biol Chem 281:1442-1448

15. Graham RM, Thompson JW, Wei J, Bishopric NH, Webster KA (2007) Regulation of bnip3 death pathways by calcium, phosphorylation, and hypoxia-reoxygenation. Antioxid Redox Signal 9:1309-1316

16. Guo K, Searfoss G, Krolikowski D, Pagnoni M, Franks C, Clark K, Yu KT, Jaye M, Ivashchenko Y (2001) Hypoxia induces the expression of the pro-apoptotic gene BNIP3. Cell Death Differ 8:367-376 
17. Gustafsson AB, Gottlieb RA (2006) Bcl-2 family members and apoptosis taken to heart. Am J Physiol Cell Physiol 292:C45-C51

18. Halestrap AP, Clarke SJ, Javadov SA (2004) Mitochondrial permeability transition pore opening during myocardial reperfusion: a target for cardioprotection. Cardiovasc Res 61:372-385

19. Hamacher-Brady A, Brady NR, Logue SE, Sayen MR, Jinno M, Kirshenbaum LA, Gottlieb RA, Gustafsson AB (2007) Response to myocardial ischemia/reperfusion injury involves Bnip3 and autophagy. Cell Death Differ 14:146-157

20. Imazu T, Shimizu S, Tagami S, Matsushima M, Nakamura $Y$, Miki T, Okuyama A, Tsujimoto Y (1999) Bcl-2/E1B 19 kDainteracting protein 3-like protein (Bnip3L) interacts with bcl-2/ $\mathrm{Bcl}-\mathrm{xL}$ and induces apoptosis by altering mitochondrial membrane permeability. Oncogene 18:4523-4529

21. Kabeya Y, Mizushima N, Yamamoto A, Oshitani-Okamoto S, Ohsumi Y, Yoshimori T (2004) LC3, GABARAP, and GATE16 localize to autophagosomal membrane depending on form-II formation. J Cell Sci 117:2805-2812

22. Kanzawa T, Zhang L, Xiao L, Germano IM, Kondo Y, Kondo S (2005) Arsenic trioxide induces autophagic cell death in malignant glioma cells by upregulation of mitochondrial cell death protein BNIP3. Oncogene 24:980-991

23. Korman EF, Blondin GA, Vail WJ, Green DE (1970) The mechanism of mitochondrial swelling: VII. The constant topology of the mitochondrial inner membrane during swelling. J Bioenerg 1:379-386

24. Koshiba T, Detmer SA, Kaiser JT, Chen H, McCaffery JM, Chan DC (2004) Structural basis of mitochondrial tethering by mitofusin complexes. Science 305:858-862

25. Kroemer G, Galluzzi L, Brenner C (2007) Mitochondrial membrane permeabilization in cell death. Physiol Rev 87:99-163

26. Kubasiak LA, Hernandez OM, Bishopric NH, Webster KA (2002) Hypoxia and acidosis activate cardiac myocyte death through the Bcl-2 family protein BNIP3. Proc Natl Acad Sci USA 99:12825-12830

27. Kubli DA, Ycaza JE, Gustafsson AB (2007) Bnip3 mediates mitochondrial dysfunction and cell death through Bax and Bak. Biochem J 405:407-415

28. Kubli DA, Quinsay MN, Huang C, Lee Y, Gustafsson AB (2008) Bnip3 functions as a mitochondrial sensor of oxidative stress during myocardial ischemia and reperfusion. Am J Physiol Heart Circ Physiol 295:H2025-H2031

29. Kuwana T, Mackey MR, Perkins G, Ellisman MH, Latterich M, Schneiter R, Green DR, Newmeyer DD (2002) Bid, Bax, and lipids cooperate to form supramolecular openings in the outer mitochondrial membrane. Cell 111:331-342

30. Landes T, Emorine LJ, Courilleau D, Rojo M, Belenguer P, Arnaune-Pelloquin L (2010) The BH3-only Bnip3 binds to the dynamin Opa1 to promote mitochondrial fragmentation and apoptosis by distinct mechanisms. EMBO Rep 11:459-465

31. Lee Y, Gustafsson AB (2009) Role of apoptosis in cardiovascular disease. Apoptosis 14:536-548

32. Lemasters JJ (2005) Selective mitochondrial autophagy, or mitophagy, as a targeted defense against oxidative stress, mitochondrial dysfunction, and aging. Rejuvenation Res 8:3-5

33. Levine B, Klionsky DJ (2004) Development by self-digestion: molecular mechanisms and biological functions of autophagy. Dev Cell 6:463-477

34. Mellor HR, Rouschop KM, Wigfield SM, Wouters BG, Harris AL (2010) Synchronised phosphorylation of BNIP3, Bcl-2, and Bcl$\mathrm{xL}$ in response to microtubule-active drugs is JNK-independent and requires a mitotic kinase. Biochem Pharmacol 79:1562-1572

35. Novak I, Kirkin V, McEwan DG, Zhang J, Wild P, Rozenknop A, Rogov V, Lohr F, Popovic D, Occhipinti A, Reichert AS, Terzic J, Dotsch V, Ney PA, Dikic I (2010) Nix is a selective autophagy receptor for mitochondrial clearance. EMBO Rep 11:45-51
36. Ong SB, Subrayan S, Lim SY, Yellon DM, Davidson SM, Hausenloy DJ (2010) Inhibiting mitochondrial fission protects the heart against ischemia/reperfusion injury. Circulation 121:20122022

37. Quinsay MN, Lee Y, Rikka S, Sayen MR, Molkentin JD, Gottlieb RA, Gustafsson AB (2010) Bnip3 mediates permeabilization of mitochondria and release of cytochrome $\mathrm{c}$ via a novel mechanism. J Mol Cell Cardiol 48:1146-1156

38. Quinsay MN, Thomas RL, Lee Y, Gustafsson AB (2010) Bnip3mediated mitochondrial autophagy is independent of the mitochondrial permeability transition pore. Autophagy 6:17-24

39. Ray R, Chen G, Vande VC, Cizeau J, Park JH, Reed JC, Gietz RD, Greenberg AH (2000) BNIP3 heterodimerizes with Bcl-2/ $\mathrm{Bcl}-\mathrm{X}(\mathrm{L})$ and induces cell death independent of a Bcl-2 homology 3 (BH3) domain at both mitochondrial and nonmitochondrial sites. J Biol Chem 275:1439-1448

40. Regula KM, Ens K, Kirshenbaum LA (2002) Inducible expression of BNIP3 provokes mitochondrial defects and hypoxiamediated cell death of ventricular myocytes. Circ Res 91: 226-231

41. Rikka S, Thomas RL, Kubli DA, Zhang X, Murphy AN, Quinsay MN, Gustafsson $\AA$ B (2010) Bnip3 impairs mitochondrial bioenergetics and stimulates mitochondrial turnover. Cell Death Differ. doi:10.1038/cdd.2010.146

42. Schwarten M, Mohrluder J, Ma P, Stoldt M, Thielmann Y, Stangler T, Hersch N, Hoffmann B, Merkel R, Willbold D (2009) Nix directly binds to GABARAP: a possible crosstalk between apoptosis and autophagy. Autophagy 5:690-698

43. Schweers RL, Zhang J, Randall MS, Loyd MR, Li W, Dorsey FC, Kundu M, Opferman JT, Cleveland JL, Miller JL, Ney PA (2007) NIX is required for programmed mitochondrial clearance during reticulocyte maturation. Proc Natl Acad Sci USA 104: 19500-19505

44. Shaw J, Zhang T, Rzeszutek M, Yurkova N, Baetz D, Davie JR, Kirshenbaum LA (2006) Transcriptional silencing of the death gene BNIP3 by cooperative action of NF-kappaB and histone deacetylase 1 in ventricular myocytes. Circ Res 99:1347-1354

45. Shaw J, Yurkova N, Zhang T, Gang H, Aguilar F, Weidman D, Scramstad C, Weisman H, Kirshenbaum LA (2008) Antagonism of E2F-1 regulated Bnip3 transcription by NF-kappaB is essential for basal cell survival. Proc Natl Acad Sci USA 105: 20734-20739

46. Smirnova E, Griparic L, Shurland DL, van der Bliek AM (2001) Dynamin-related protein Drp1 is required for mitochondrial division in mammalian cells. Mol Biol Cell 12:2245-2256

47. Sowter HM, Ratcliffe PJ, Watson P, Greenberg AH, Harris AL (2001) HIF-1-dependent regulation of hypoxic induction of the cell death factors BNIP3 and NIX in human tumors. Cancer Res 61:6669-6673

48. Syed F, Odley A, Hahn HS, Brunskill EW, Lynch RA, Marreez Y, Sanbe A, Robbins J, Dorn GW II (2004) Physiological growth synergizes with pathological genes in experimental cardiomyopathy. Circ Res 95:1200-1206

49. Twig G, Elorza A, Molina AJ, Mohamed H, Wikstrom JD, Walzer G, Stiles L, Haigh SE, Katz S, Las G, Alroy J, Wu M, Py BF, Yuan J, Deeney JT, Corkey BE, Shirihai OS (2008) Fission and selective fusion govern mitochondrial segregation and elimination by autophagy. Embo J 27:433-446

50. Vande VC, Cizeau J, Dubik D, Alimonti J, Brown T, Israels S, Hakem R, Greenberg AH (2000) BNIP3 and genetic control of necrosis-like cell death through the mitochondrial permeability transition pore. Mol Cell Biol 20:5454-5468

51. Wei MC, Zong WX, Cheng EH, Lindsten T, Panoutsakopoulou V, Ross AJ, Roth KA, MacGregor GR, Thompson CB, Korsmeyer SJ (2001) Proapoptotic BAX and BAK: a requisite 
gateway to mitochondrial dysfunction and death. Science 292:727-730

52. Yasuda M, Theodorakis P, Subramanian T, Chinnadurai G (1998) Adenovirus E1B-19K/BCL-2 interacting protein BNIP3 contains a $\mathrm{BH} 3$ domain and a mitochondrial targeting sequence. J Biol Chem 273:12415-12421

53. Yoon Y, Krueger EW, Oswald BJ, McNiven MA (2003) The mitochondrial protein hFis1 regulates mitochondrial fission in mammalian cells through an interaction with the dynamin-like protein DLP1. Mol Cell Biol 23:5409-5420

54. Yurkova N, Shaw J, Blackie K, Weidman D, Jayas R, Flynn B, Kirshenbaum LA (2008) The cell cycle factor E2F-1 activates
Bnip3 and the intrinsic death pathway in ventricular myocytes. Circ Res 102:472-479

55. Yussman MG, Toyokawa T, Odley A, Lynch RA, Wu G, Colbert MC, Aronow BJ, Lorenz JN, Dorn GW II (2002) Mitochondrial death protein Nix is induced in cardiac hypertrophy and triggers apoptotic cardiomyopathy. Nat Med 8:725-730

56. Zhang J, Ney PA (2009) Role of BNIP3 and NIX in cell death, autophagy, and mitophagy. Cell Death Differ 16:939-946

57. Zong WX, Lindsten T, Ross AJ, MacGregor GR, Thompson CB (2001) BH3-only proteins that bind pro-survival Bcl-2 family members fail to induce apoptosis in the absence of Bax and Bak. Genes Dev 15:1481-1486 